\title{
Use of budesonide Turbuhaler® in young children suspected of asthma
}

\author{
H. Bisgaard*, S. Pedersen**, K. Nikander ${ }^{\dagger}$
}

Use of budesonide Turbuhaler ${ }^{\circledR}$ in young children suspected of asthma. H. Bisgaard, S. Pedersen, K. Nikander. CERS Journals Ltd 1994.

ABSTRACT: The question addressed in this study was the ability of young children to use a dry-powder inhaler, Turbuhaler®.

One hundred and sixty five children suspected of asthma, equally distributed in one year age-groups from 6 months to 8 yrs, inhaled from a Pulmicort Turbuhaler ${ }$, $200 \mu \mathrm{g}$ budesonide. dose $^{-1}$, through a filter. The amount of drug trapped by the filter was used as a measure of drug released to the patient. None of the children had prior experience in the use of a Turbuhaler ${ }^{\circledR}$, but they were instructed carefully, together with their parents, in the clinic.

The median dose released revealed an age-dependent increase, with a considerable scatter. Accordingly, the dose delivered could not be predicted in these young children. The limitation to effective use in young children appeared to be lack of sufficient co-operation, rather than physical limitations, as even some very young children appeared to obtain a sufficient activation of the device. It is likely that repeated training at home may improve these findings.

In conclusion, our results indicate that dry-powder inhalers are not reliable in all circumstances for treatment of young children, and that careful and repeated tuition is required if such devices are to be used.

Eur Respir J., 1994, 7, 740-742.

\author{
*Dept of Paediatrics, State University Hos- \\ pital, Rigshospitalet, Copenhagen, Denmark. \\ **Dept of Paediatrics, Kolding County \\ Hospital, Denmark. †Clinical Research \& \\ Development, Astra Draco AB, Lund, \\ Sweden. \\ Correspondence: H. Bisgaard \\ Dept of Paediatrics \\ State University Hospital \\ Rigshospitalet \\ Copenhagen \\ Denmark \\ Keywords: Aerosol treatment \\ asthma \\ dry powder inhaler \\ Turbuhaler ${ }^{\circledR}$ \\ young children
}

Received: January 211994

Accepted for publication February 91994
The dry-powder inhalers, such as Turbuhaler® and Diskhaler ${ }^{\circledR}$, have been developed for use in adults and schoolchildren with bronchial asthma. However, in our clinical experience, an increasing number of preschool children suspected of asthma have been provided with Turbuhaler ${ }^{\circledR}$ or Diskhaler ${ }^{\circledR}$, even though their ability to use these devices properly has not been documented. The generation of aerosol from all available dry-powder inhalers is inspiratory flow-dependent. Generation of an inspiratory flow sufficient to activate the dry-powder inhaler is dependent not only on physical limitations to flow but, probably more importantly, on the ability of the patient to co-operate.

In order to investigate the capability of children, with suspected asthma, younger than 8 years to use a drypowder inhaler, we designed a study using the amount of budesonide leaving the Pulmicort Turbuhaler ${ }^{\circledR}$ during inhalation as a measure of a child's ability to use this dry-powder inhaler.

\section{Patients and methods}

The study was accepted by the local Ethics Committee. We selected 165 children from an out-patient clinic in Copenhagen, equally distributed within one year agegroups from 6 months to $8 \mathrm{yrs}$, and studied them in randomized order. The children all suffered from recurrent wheezing, but were free of symptoms on the study day. They all had a clinical response to inhalation of $\beta_{2}$-agonist. All children inhaled from a Pulmicort Turbuhaler ${ }^{\circledR}, 200 \mu \mathrm{g} \cdot \mathrm{dose}^{-1}$. None of the children had previously used a dry-powder inhaler. The children and their parents were carefully instructed in the inhalation technique by the clinic's instruction group. A placebo Turbuhaler® was used by the nurse to demonstrate the inhalation technique. If obvious errors were made during the inhalation manoeuvre a new attempt was allowed, after changing the filter equipment.

A filter (Respirgard-II MQ-303 filter, Marquest Medical Products, USA) was interposed in its holder between the Turbuhaler® and the patient's mouth, in order to collect the dose of budesonide from the Pulmicort Turbuhaler®. The dose of budesonide released was almost completely retained in the filter, as less than $1 \%$ of this dose could be collected on a second filter placed after the first filter in pilot studies (data on file). The pressure drop over the filter equals approximately $6 \mathrm{mmH}_{2} \mathrm{O}$ at a flow of $60 l \cdot \mathrm{min}^{-1}$. The volume of airspace between the Turbuhaler® mouthpiece and this filter was $46 \mathrm{ml}$, which equals the maximal volume necessary to inhale before the aerosol reaches the filter (fig. 1). The children less than 48 months old used a tightly fitting facemask (Astra Meditec, Sweden) attached to the mouthpiece of the filter, in order 


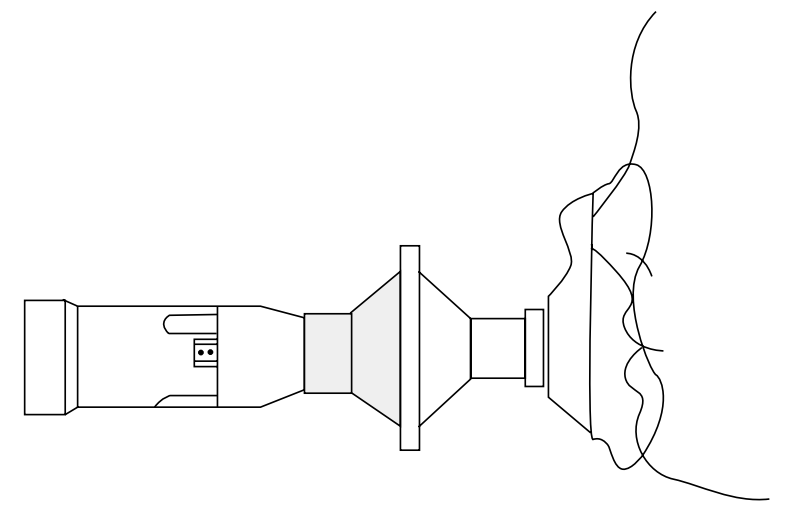

Fig. 1. - The filter with facemask. The volume necessary to move the dry-powder aerosol from the Turbuhaler ${ }^{\circledR}$ to the filter is approximately $46 \mathrm{ml}$ (dotted area). The presence of the facemask obviously does not add any dead space with respect to collection of the aerosol.

to avoid air entainment through the nose and around the mouthpiece. The children in the age range 48-96 months inhaled directly from the mouthpiece of the filter.

The same Turbuhaler ${ }^{\circledR}$ was used for all administrations, but the filter, filter-holder and mouthpiece were replaced between each treatment. The filter and filterholder were washed with ethanol to dissolve retained drug. The amount of trapped budesonide was determined by liquid chromatography using internal standard. The relative standard deviation of the assay was $3 \%$. The total amount of drug released to the child was estimated by addition of the doses on both the filter and filterholder.

\section{Results}

An age-dependent increase in the dose of budesonide delivered was found (fig. 2). Children older than 6 yrs of age obtained a median of $55 \%$ of the nominal dose, and children younger than 3 yrs of age obtained a median dose of $9 \%$. The age-dependency of the dose inhaled was obvious in the children older than 48 months, who all inhaled from the mouthpiece. Younger children

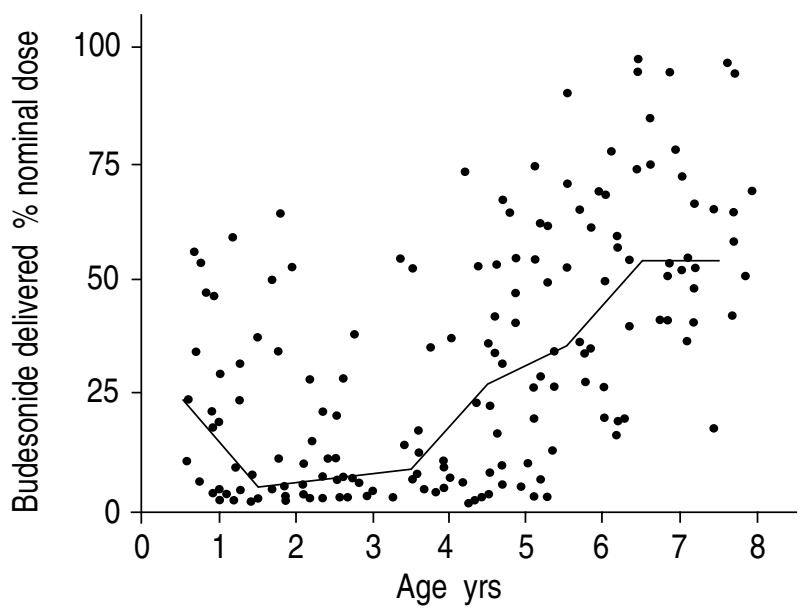

Fig. 2. - Doses of budesonide trapped on filter equipment after inhalation by children from Turbuhaler ${ }^{\circledR}$, expressed as percentage of nonimal dose $\left(200 \mu \mathrm{g} \cdot \mathrm{dose}^{-1}\right)$. Median values are shown by the line. A further increase in dose-delivery can be expected in children above 8 yrs of age [1]. obtained a low dose, with no obvious age-dependency, but a considerable scatter.

The dose of budesonide trapped on the filter equipment showed a considerable variation; this included some very young children getting doses equivalent to the median dose in 6-7 year old children, and others getting almost nothing. The coefficient of variation for dose to all children was $87 \%$.

\section{Discussion}

We found that the filter dose from Pulmicort Turbuhaler® showed a considerable scatter and an age-dependent increase in children from 4-8 yrs of age. Younger children obtained a very low dose, though with a considerable scatter. The children younger than 48 months inhaled from the Turbuhaler ${ }^{\circledR}$ through a facemask, in order to direct as much as possible of the inspiratory flow through the Turbuhaler® device. Without the facemask, only a few of the youngest children would be able to perform an inspiration from the mouthpiece without air entainment through the nose and around the mouthpiece. Inspiration through the nose may still have occurred within the facemask in some of the children. This may explain some of the cases of poor peak inspiratory flow observed, as the nasal airway resistance limits the flow obtainable, yet this situation represents the best obtainable.

The large variation in dose obtained is probably not solely due to a physical limitation of the inspiratory flow, as a number of very young children obtained doses equal to those obtained by $6-8$ year old children. The ability of the child to co-operate in the generation of the peak inspiratory flows and volumes required is equally important, and is not possible to predict.

It has previously been reported that an optimum clinical response to terbutaline inhaled from Bricanyl Turbuhaler ${ }^{\circledR}$ requires an inspiratory flow of at least $30 l \cdot \mathrm{min}^{-1}[2]$. In children younger than 6 yrs, the ability to perform a peak inspiratory flow of this magnitude varied markedly, and, therefore, supports the present findings of highly variable doses inhaled by the young children.

The tidal volume of young children is about 6-7 $\mathrm{ml} \cdot \mathrm{kg}^{-1}$ body weight, which in most infants older than 6 months of age is more than the volume between Turbuhaler® and the filter (fig. 1). Even tidal inhalation will, therefore, move sufficient volume of air for any aerosol to reach the filter. The resistance of the filter is less than the inspiratory pressure that may be generated by infants. It is, therefore, reasonable to assumed that the trapped dose reflects fairly well the dose released to the child. Certainly, this trapped dose cannot be extrapolated to the dose of drug that would have reached the lower airways, as the particle size is unknown. Particles contain fractions of the dose proportional to the cube of the radii. A considerable fraction of the dose collected could, therefore, be contained in a few large particles, which would not reach the lower airways. Furthermore, the deaggregation of micronized budesonide in the inhalation channel is impaired at low flow rates. Thus, the dose 
of respirable budesonide particles may be more severely affected by the poor performance of some of the children than was reflected by the total dose of drug collected on the filter. The total dose of drug may, therefore, be a poor measure of comparison between different inhaler devices of unknown flow-dependent particle profile characteristics; however, the method is useful for studying the age-dependency of the dose delivery from a single device. The presently illustrated age-dependent decrease of the dose released to the child, accordingly reflects the minimum drop in dose reaching the lower airways.

It was the aim of our study to investigate the average child, who did not have any previous experience in the use of Turbuhaler®. The children were given instruction, which would match the instruction given in most clinics. Repeated instructions and training in the inhalation technique may improve the performance of these preschool children. It is, therefore, not possible from the present study to define an age limit at which children can safely use dry-powder inhalers and below which it is impossible. In many cases, even a young child may appear welltreated with glucocorticosteroid or $\beta_{2}$-agonist from a dry-powder inhaler. However, even though the more compliant child may learn how to perform a correct inhalation manoeuvre through the dry-powder inhaler, the same child may not be able to live up to this standard when he is ill, tired and whimpering, and certainly not during acute exacerbations. It is, therefore, preferable to treat young children with devices not requiring cooperation, such as inhalation of a metered-dose inhaler (MDI) with a spacer device or a jet-nebulizer.

The dry-powder inhalers, such as Turbuhaler ${ }^{\circledR}$ and Diskhaler ${ }^{\circledR}$, have been designed for use in adults and school-children and are not recommended for use in children below the age of 5 yrs (Turbuhaler ${ }^{\circledR}$ ), or 3 yrs (Diskhaler $\left.{ }^{\circledR}\right)$. The present data confirm that Turbuhaler® should be used with caution in preschool children, as the devices cannot be relied upon to deliver predictable doses of drug in young children under all circumstances. Similar precautions may be considered in the use of the Diskhaler®. We, accordingly, feel that our results may serve to warn against indiscriminate use of dry-powder inhalers in the treatment of young children.

\section{References}

1. Nikander K, Bisgaard H, Pedersen S. Output of budesonide from three different inhalation systems used by patients with bronchial asthma. 4th International Symposium: Deposition and Clearance of Aerosol in the Human Respiratory Tract. August 1992, Vienna, Austria, (Abstract).

2. Pedersen S, Hansen OR, Fuglsang G. Influence of inspiratory flow upon the effect of a Turbuhaler®. Arch Dis Child 1990; 65: 308-319. 